\title{
Dispositivos de
}

conmutación óptica
en redes de nueva
generación

\section{Optical switching devices in next generation network}

\section{Resumen}

Este documento muestra las funcionalidades de dos tipos de conmutadores ópticos: OXC y OADM, utilizando una herramienta de software que simula el comportamiento de cada uno de estos dispositivos desarrollada en Matlab. Se realiza un análisis comparativo entre los diferentes tipos de interruptores ópticos enfatizando en los interruptores ópticos MEMS (2-D y 3D). Se muestran las diferentes configuraciones para las matrices de conmutación en una sola celda, junto con sus principales características, también se muestran los principales problemas de diseño en relación con las cualidades del interruptor óptico: el rendimiento óptico y las funcionalidades electromecánicas. En el caso del diseño óptico, se analiza la pérdida de inserción inducida por la desalineación de la fibra, la reflexión de Fresnel y aspectos relacionados con el micro-espejo, incluido el material de recubrimiento, el grosor, la rugosidad de la superficie, el efecto del espejo en diferentes longitudes de onda y la pérdida de la polarización-dependiente (PDL).

\section{Palabras clave}

Interruptor óptico MEMS, interruptor óptico OADM, interruptor óptico OXC.

Ingeniero Electrónico y Magíster en Ciencias de la Información y las Comunicaciones, Universidad Distrital Francisco José de Caldas. Docente y Director del Grupo investigación GITUD, Universidad Distrital. E-mail: egaona@udistrital.edu.co

** Ingeniero de Sistemas y Magíster en Ciencias de la Información y las Comunicaciones, Universidad Distrital Francisco José de Caldas. Docente y Director del Grupo investigación GIIRA, de la Universidad Francisco José de Caldas. E-mail: pagaonag@udistrital.edu.co

*** Ingeniero de Sistemas, Universidad Distrital Francisco José de Caldas. Doctor en Informática de la Universidad de Oviedo (España) Magíster en Ciencias de la Información y las Comunicaciones, Universidad Distrital Francisco José de Caldas. Docente e Investigador del Grupo investigación GIIRA, Universidad Distrital Francisco José de Caldas. E-mail: cemontenegrom@udistrital.edu.co 


\section{Abstract}

This paper aims to give a clear view of the features of two types of optical switches, OXC and OADM's, by developing software that simulates the behavior of each of these devices using the Matlab tool. The document begins with a comparative study between different types of optical switches and optical switches with emphasis on MEMS (2-D and 3D), after present the different settings for routing switchers in a single cell, together with their main characteristics. It covers the main design issues in relation to two characteristics of the optical switch, optical performance and electromechanical features. For the optical design, we examine the insertion loss induced by misalignment of the fiber, the Fresnel reflection and issues related to the micro-mirror, including the coating material, thickness, surface roughness, the effect of mirror at different wavelengths and the loss of polarization-dependent (PDL).

\section{Keywords}

\section{Optical Switch MEMS, Optical Switch OADM and Optical Switch OXC.}

\section{Introducción}

Debido a los avances tecnológicos en fibra óptica y en particular a la fabricación de dispositivos ópticos se ha incrementado la capacidad de transmisión de información en los sistemas de comunicación. Así como la conmutación eléctrica sustituyó a los relés mecánicos del pasado, la conmutación óptica está a punto de reemplazar los módulos de la conmutación eléctrica existentes en la actualidad. Un claro ejemplo de ello, es una red totalmente óptica (AON), en donde los conmutadores ópticos seleccionan las direcciones de la señal, adicionan o eliminan información, protegen las redes, etc. Todas estas funciones se pueden realizar con los tradicionales conmutadores eléctricos, que luego de convertir la señal óptica, la convierte en una señal eléctrica para posteriormente convertirse nuevamente en una señal óptica para transmisiones de otro tipo [1].

La conmutación óptica es de vital importancia, ya que la conversión de las señales ópticas a electrónicas es un cuello de botella crítico en las redes totalmente ópticas AON, esto se debe a la enorme cantidad de información que tiene que conmutarse a través de varios nodos. Por lo tanto, la conmutación óptica es un desarrollo prometedor y una pieza clave en las redes ópticas dinámicamente reconfigurables [1].

Como consecuencia de los estrictos requerimientos del ancho de banda, los interruptores MEMS de fibra óptica cada vez son más importantes en cuanto a la comunicación óptica. El diseño de estos dispositivos MEMS plantea muchos desafíos como los problemas de diseño en relación con dos características principales del interruptor óptico: el rendimiento óptico, que está condicionado por la pérdida de inserción inducida causada por los tres tipos de desalineación de la fibra, la reflexión de Fresnel, y las características electromecánicas. El sistema óptico determina de igual forma las propiedades ópticas de los espejos, teniendo en cuenta el material de recubrimiento, el grosor, la rugosidad de la superficie, el efecto del espejo en diferentes longitudes de onda y la pérdida de la 
polarización dependiente (PDL por sus siglas en inglés), parámetros que establecen el rendimiento final del interruptor [3].

La herramienta de simulación desarrollada analiza el comportamiento de tres dispositivos de conmutación fotónica, además permite pronosticar fallas en cualquier entorno adverso donde funcione, reduciendo eventos indeseables y corregirlos a tiempo de manera que se logre con éxito los proyectos que manejen conmutación fotónica en redes ópticas.

\section{Interruptores ópticos}

El conmutador óptico es un mecanismo importante en un sistema de comunicación de fibra óptica, desarrollándose con el uso de diferentes técnicas, como la óptica de MEMS, métodos termo-ópticos, amplificadores ópticos de semiconductores (SOA), cristales líquidos, electroholografía, electro-ópticos LiNbO3, guía de onda conmutable electrónicamente (Bragg grating), acusto-ópticos y los conmutadores opto-ópticos [2], [16].

Estos mecanismos tienen sus ventajas y sus desventajas, en términos de las características de rendimiento (pérdida de inserción, velocidad de conmutación, diafonía, pérdida dependiente de la polarización, tasa de bits, transparencia del protocolo y ancho de banda de operación), requisitos de red (multicast, dimensiones de los dispositivos de conmutación y escalabilidad), y requerimientos del sistema (estabilidad, repetibilidad, consumo de energía y costo) [2].

En la tabla 1, se enumeran las características de rendimiento promedio más importantes asociados con cada uno de los tipos de interruptores ópticos, con el propósito de una comparación general. Sin embargo, la combinación de las diferentes características de interruptores, determina la posible aplicación de este tipo de interruptor [5].

\begin{tabular}{|c|c|c|c|c|}
\hline Tipo de interruptor & Velocidad de conmutación & Diafonía & Tamaño & $\begin{array}{c}\text { Voltaje/Potencia de } \\
\text { disipación }\end{array}$ \\
\hline MEMS óptico & Sub-ms a ms & $\leq-30 \mathrm{Db}$ & $\mathrm{mm} \times \mathrm{mm}$ & $\leq 50 \mathrm{~V}$ \\
\hline \hline $\begin{array}{c}\text { Termo-óptico } \\
\text { (MZl Interferómetro) }\end{array}$ & $\mu$ s a ms & $>-30 \mathrm{Db}$ & $\mathrm{mm} \times \mu \mathrm{m}$ & $90 \mathrm{~mW}$ \\
\hline \hline Termo-óptico (DOS) & $<5 \mathrm{~ms}$ & $\leq-30 \mathrm{Db}$ & $\mathrm{mm} \times \mathrm{mm}$ & $250 \mathrm{Mw}$ \\
\hline \hline Electro-óptico (LiNb03) & $5 \mathrm{~ns}$ & $<-45 \mathrm{~dB}$ & $3-\mathrm{in}$ & $18 \mathrm{~V}$ \\
\hline \hline Basados en SOA & $200 \mathrm{ps}$ & $\leq-12 \mathrm{~dB}$ & $\mathrm{~mm} \times \mathrm{mm} \times \mathrm{mm}$ & $200 \mathrm{~mA}$ \\
\hline \hline Cristal líquido & $\mathrm{Ms}$ & $\leq-35 \mathrm{~dB}$ & $\mathrm{~mm} \times \mathrm{mm} \times \mathrm{mm}$ & Muy Bajo \\
\hline \hline Electroholográfico & $<10 \mathrm{~ns}$ & Muy Bajo & $\mathrm{mm} \times \mathrm{mm}$ & Alto Voltaje \\
\hline \hline Acusto-óptico & $300 \mathrm{~ns}$ & $32 \mathrm{Db}$ & $\mathrm{cm}$ & $200 \mathrm{Mw}$ \\
\hline \hline
\end{tabular}

Tabla 1. Comparación del rendimiento de los diferentes interruptores ópticos.

Se puede observar claramente que los MEMS ópticos y los interruptores termo-ópticos son los más apropiados para ser usados en dispositivos portátiles de bajo costo, como lo son: optical cross connect (OXC) y optical add/drop multiplexers (OADM). Mientras que los interruptores electro-ópticos y acusto-ópticos tienen el potencial para la conmutación 
de paquetes a una alta velocidad y de manera altamente confiable, pero el dispositivo presenta una desventaja, ya que puede ser voluminoso y de alto costo [3].

\section{A. Interruptores ópticos MEMS}

Un interruptor óptico MEMS representa la integración de funcionalidades ópticas, eléctricas y mecánicas en un solo chip, el cual, mediante el uso de un espejo o una membrana, físicamente modifica la propagación de un rayo de luz [25]-[27]. Además, muestra una ventaja gigantesca al lograr: bajos cross talks, altas tasas de extinción, independencias en los formatos de los datos y las velocidades, insensibilidad en las longitudes de las ondas y sobre todo, fácil integración a gran escala [3], [17], [24], [27].

El de tipo MEMS consta de tres partes principales: un actuador de peine, una viga doblada de suspensión y un micro-espejo [3], [17]. Las tres partes principales están conectadas por una red troncal, en donde el surco $b$ se fabrica para el montaje de la fibra óptica y los surcos a, $c$ y $d$ se insertan y se fijan en estas ranuras. Como se muestra en la Figura 1, [6].

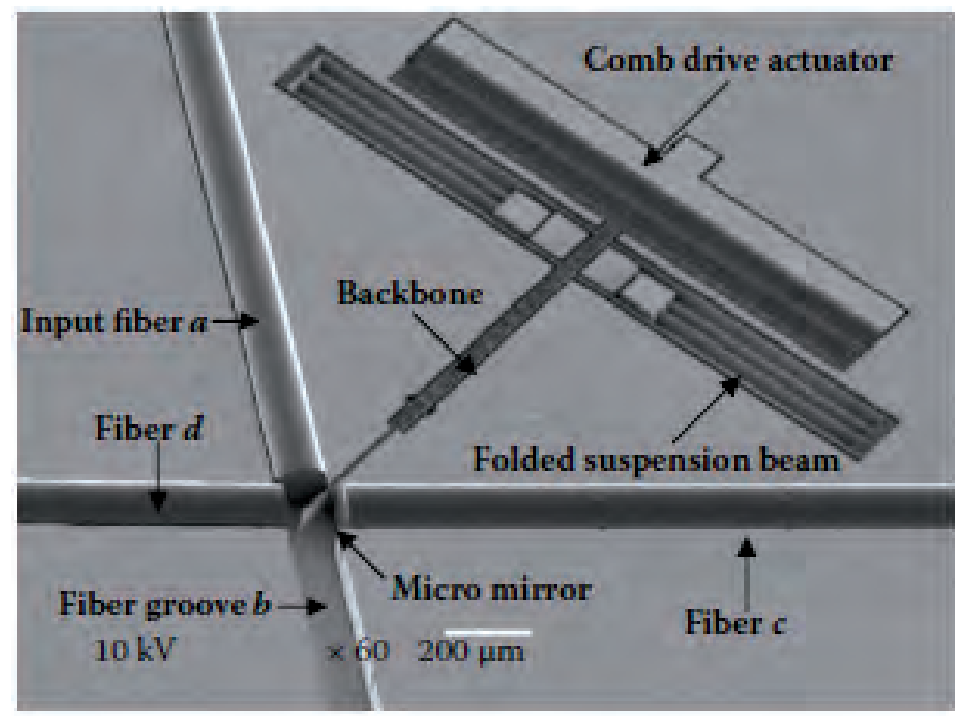

Figura 1. Vista general del interruptor MEMS.

- El actuador de peine se utiliza para cambiar la posición del micro-espejo dentro y fuera de la trayectoria del haz.

- La viga doblada de suspensión se utiliza para equilibrar la fuerza electrostática inducida por la unidad del peine cuando la tensión externa se aplica al actuador de peine.

La fuerza elástica generada por el haz impulsará al interruptor a su posición original cuando la tensión aplicada es retirada. El micro-espejo como otro componente clave del interruptor, se utiliza para dirigir el haz incidente. Cuando el voltaje adecuado se aplica al actuador, el micro-espejo es expulsado de la trayectoria del haz de la unidad de peine, y el haz incidente pasará a través de la fibra de entrada a la salida de la fibra $b$ directamente. Cuando la tensión se libera, la fuerza electrostática se descarga mientras que la fuerza 
elástica de la viga de suspensión sigue existiendo, la unidad conduce al micro-espejo a la posición original, cambiando el interruptor al estado cruz. Los haces incidentes de las ramas a y $c$ se reflejaran por el micro-espejo $a, d$ y $b$, respectivamente, como resultado de la alta reflectividad de ambos lados del micro-espejo [6], [17].

\section{B. Interruptores ópticos MEMS en 2-D}

El interruptor óptico MEMS 2-D tiene sus propias ventajas sobre el interruptor de espejo 3-D, tal como el bajo costo y la fabricación de manera sencilla. Además, la fiabilidad y repetitividad son mejores. A pesar de que la escalabilidad es un problema, el interruptor óptico MEMS 2-D tiene la ventaja de las matrices pequeñas y medianas. En la actualidad el mercado está dominado por matrices pequeñas $(2 \times 2$ y $4 \times 4)$ y medianas (por ejemplo, $16 \times 16$ ), el interruptor $2-D$ se puede utilizar en matrices con un tamaño de hasta $64 \times 64$ [4], [18].

Para la celda en el interruptor torsional MEMS 2-D, un espejo altamente reflectivo es impulsado a realizar un movimiento de torsión sobre un eje determinado (o sobre varios ejes). El micro-espejo está suspendido sobre el sustrato por micro-vigas a lo largo de la dirección axial, y estos a su vez están soportados por los anclajes en los extremos. Los electrodos opuestos se encuentran bajo el micro-espejo, de esta manera, el micro-espejo puede ser utilizado para girar alrededor del eje de la viga por la fuerza electrostática [4], [18].

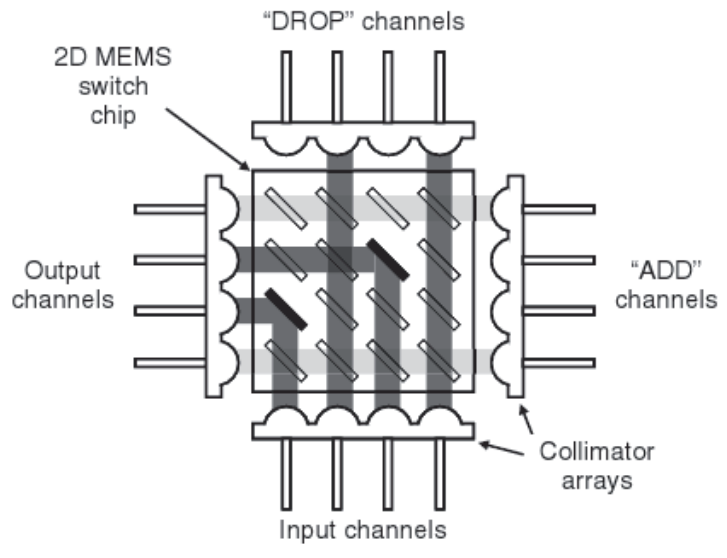

Figura 2. MEMS 2-D.

Este tipo de interruptor óptico se desarrolla utilizando ya sea un sustrato SOI o un plano de sustrato de silicio. Existen dos estados diferentes: cuando el espejo está en la trayectoria del haz, la luz incidente es reflejada hacia la salida ortogonal, realizando el estado cruz y cuando el espejo se retira de la trayectoria del haz, la luz incidente puede viajar a través de las fibras contrarias de salida, realizando el estado barra [7].

\section{Interruptores ópticos MEMES en 3-D}

El interruptor óptico 3-D MEMS es mejor ejemplificarlo por los OXC, ya que se pueden escalar a miles de puertos con baja pérdida y alta uniformidad. Su micro-espejo se puede 
inclinar a lo largo de dos ejes ortogonales, cuando se aplica un voltaje adecuado a los electrodos fijos y móviles. Los inconvenientes críticos en este tipo de configuración son: (1) los mecanismos complejos de retroalimentación que son necesarios para la posición de los espejos; (2) los haces de las fibras de entrada y salida deben ser alineados; y (3) los procesos de producción son complicados y costosos. La fabricación de este tipo de interruptor óptico MEMS es muy complejo, ya que requiere de sofisticados mecanismos de control analógico e intrincados empaquetamientos para conectar los cables a los electrodos [8], [14], [15].

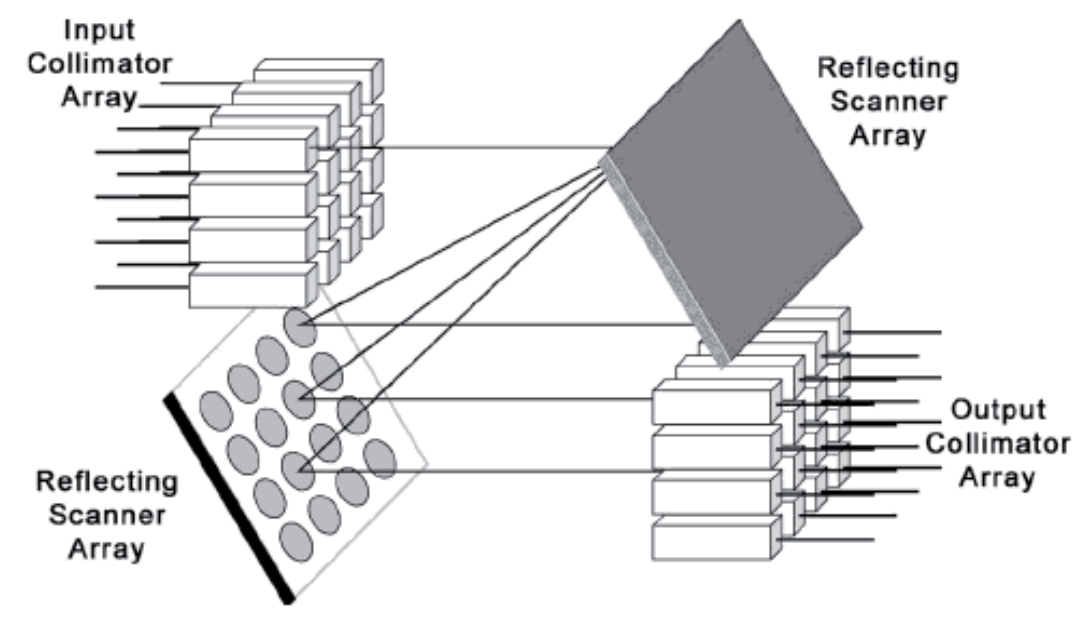

Figura 3. MEMS 3-D.

\section{Conmutador óptico OADM}

Con el desarrollo de sistemas DWDM (Multiplexación por longitud de onda densa), la eficiencia en el uso del ancho de banda de la fibra se vuelve imperativa [19]. El OADM, tiene la capacidad de añadir o retirar selectivamente un canal individual o un subconjunto de longitudes de onda del sistema de transmisión, sin una regeneración completa opto-electrónica de todas las longitudes de onda, y permite manipular el tráfico sobre la base de la longitud de onda en la capa óptica [6], [19]-[21].

Los OADM se pueden dividir en dos categorías, las cuales son estática y dinámica (reconfigurable). La primera permite a los canales predeterminados ser adicionados o retirados, mientras que la segunda puede proporcionar mucha más flexibilidad, ya que permite que los canales que se añadan o que se retiren sea de forma dinámica [6], [19]-[21].

Actualmente existen varias propuestas en el mercado de conmutadores OADM's reconfigurables en donde las redes de Bragg Grating (FBG), se configuran mediante la variación periódica de su índice de refracción a través de diferentes mecanismos y de cristal líquido ferroeléctrico (FLC) en cascada, proporcionando una rápida selección y conmutación de canales add/drop. La tecnología de guía de onda presenta una mejora significativa en la tecnología de integración de OADM, ya que es difícil de ensamblar los diferentes componentes de sustrato en un chip monolítico [22]. 
Los interruptores ópticos MEMS y de cristal líquido (LC), son dos nuevas tecnologías para la integración de OADM reconfigurables. Los interruptores ópticos MEMS son muy pequeños, por ello, cientos de ellos pueden ser colocados en el mismo espacio, son mucho menos costosos que sus homólogos y se mejora la precisión, en consecuencia, cientos de dispositivos pueden ser elaborados en un solo plano en un proceso de IC. Los micro-espejos de inclinación y válvulas de luz se han desarrollado y aplicado a los OADM reconfigurables [6].

El interruptor óptico MEMS 2x2 es un componente importante que mejora el rendimiento de los OADM reconfigurables [23]. Los módulos MEMS de fibra óptica 2x2 basados en actuadores fueron fabricados sobre un sustrato SOI. El proceso de fabricación es sencillo debido a la tecnología SOI, pero la hoja del material es de un alto costo. Debido a esto, recientemente un nuevo proceso de fabricación DRIE en una hoja de silicio se ha desarrollado, el cual evita el efecto de fricción estática y el hacer muescas del proceso basado en SOI, por lo tanto reduce el costo de fabricación de la unidad del interruptor óptico MEMS $2 \times 2[6]$.

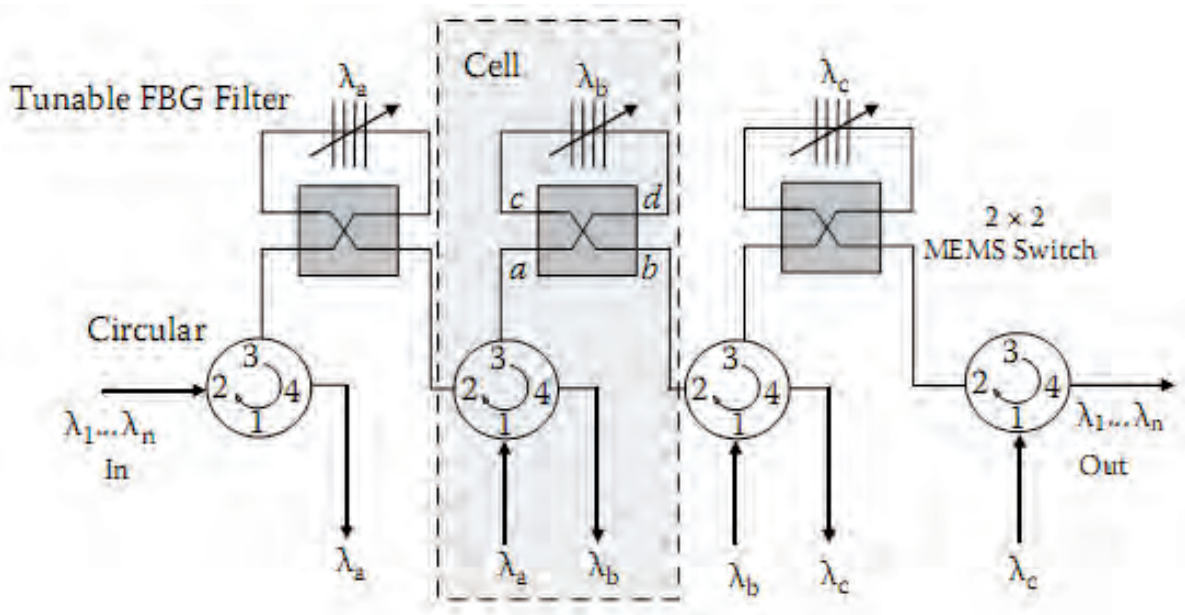

Figura 4. Construcción del interruptor óptico OADM.

Cada celda se compone de una pieza de un interruptor óptico MEMS 2x2, un OC de cuatro puertos y un FBG sintonizable cuya longitud de onda es el Bragg central, es ajustable al igualar la señal DWDM $\lambda$ i. El FBG sintonizable se inserta en las ranuras de las fibras del interruptor. De esta manera, el empalme de fibra-fibra o fibra-guía de onda no es necesario [6].

Cuando el haz multicanal lanza las señales a través del puerto dos del OC de cuatro puertos, estas van a ir a la rama a del interruptor $2 \times 2$ al puerto tres del OC. Cuando el interruptor está en el estado barra, todas las señales pasan a través del interruptor a la rama $b$, y en este caso, ningún canal será retirado en esta celda. Cuando el interruptor está en estado cruz, el haz incidente de la rama a se dirige al FBG sintonizable a través de la rama d del interruptor. Este haz alimenta al filtro sintonizable y una longitud de onda es seleccionada por el sintonizador FBG. El canal seleccionado se refleja de vuelta al puerto tres del OC y entonces sale por el puerto cuatro del OC. 
Los otros canales que no coinciden en el centro de la longitud de onda del FBG pasan a través de la rejilla a la rama c y son dirigidos por el interruptor óptico de 2x2, a la celda posterior a través de la rama $b$. La longitud de onda que es retirada por la celda anterior, es utilizada en esta celda mediante la adición de una nueva señal transportadora por la misma longitud de onda desde el puerto uno del OC. Estas longitudes de onda que pasan por el canal, continuarán su propagación a las celdas posteriores para futuras adiciones y retiradas [4].

La primera y última celda tienen la misma estructura como se describió anteriormente, pero hay una ligera diferencia en la aplicación. La primera celda no tiene ninguna responsabilidad para agregar cualquier canal. Por lo tanto, el puerto uno de la OC no se utiliza. El último de los cuatro puertos del OC, sólo es responsable de la adición de un canal de longitud de onda que ha sido retirado en la celda anterior. Por lo tanto, el puerto tres no se utiliza. Sin embargo, proporciona una mayor flexibilidad para colocar más celdas en cascada. Sólo uno de ellos puede someterse a un proceso add/drop a la vez. Este tipo de OADM es más flexible y reconfigurable que el OADM estático convencional, incluso aunque el primero todavía no es totalmente flexible. La actualización del sistema se realiza colocando en cascada más celdas sin reemplazar los componentes existentes [4].

\section{Características ópticas y comportamiento de los dispositivos de conmutación OXC y OADM}

Las siguientes propiedades ópticas están inmersas en el funcionamiento de cada uno de los interruptores ópticos OXC y OADM.

\section{A. Pérdidas de inserción}

El haz óptico siempre se transmite a lo largo del camino como se muestra en la Figura 5,

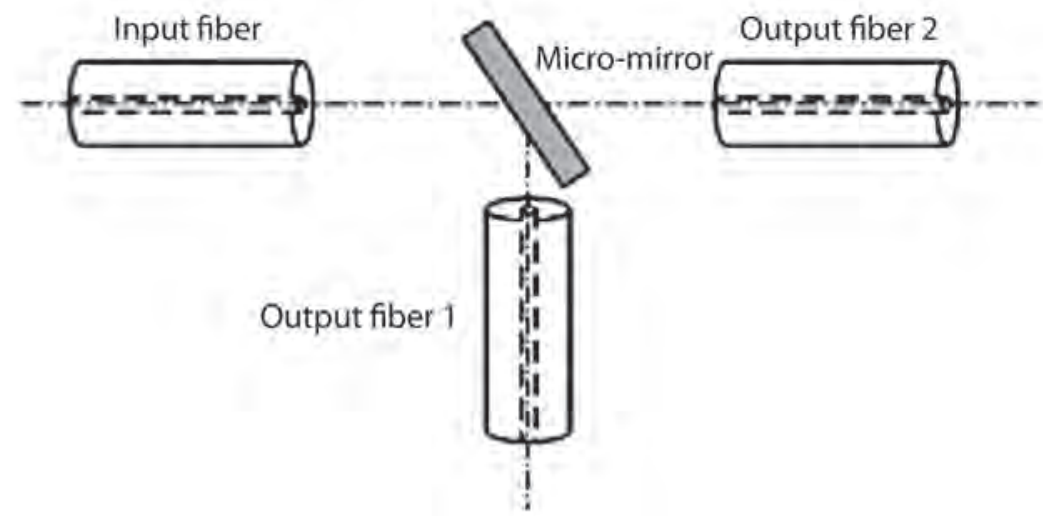

Figura 5. Esquemático del camino de un haz en un conmutador óptico.

La señal proviene de la fibra de entrada al micro-espejo y sale a la fibra de salida uno o a la fibra de salida dos, dependiendo de la posición del micro-espejo. En el estado barra, el canal de salida dos será seleccionado sin cambiar la dirección del haz. Por otra parte, para redirigir la señal de entrada al canal uno, el espejo vuelve a dirigir la trayectoria del 
haz, al mismo tiempo, induciendo una pérdida adicional. Por lo tanto, la distribución de potencia del haz, la rugosidad de la superficie del espejo, la verticalidad del espejo, y las propiedades del material del espejo son las principales causas de pérdida de potencia óptica en el sistema [9].

\section{B. Pérdida por acoplamiento de la fibra}

La distribución de campo del modo fundamental de la fibra monomodo (SMF), puede ser bien aproximada por una función de Gauss [10]. La expresión empírica siguiente describe el ancho $\omega 0$ del haz Gaussiano:

$$
\omega_{0}=\left(0.65+\frac{1.619}{V^{3 / 2}}+\frac{2.879}{V^{6}}\right) \cdot a
$$

Donde $\omega_{0}$, es el ancho del haz de Gauss, a es el radio del núcleo, y $\vee$ es el parámetro de la guía de onda dado en la ecuación. El haz se desviará cuando sale de la fibra y entra en un espacio libre porque no hay reflexión total [10]. El perfil del haz se muestra en la Figura 6.

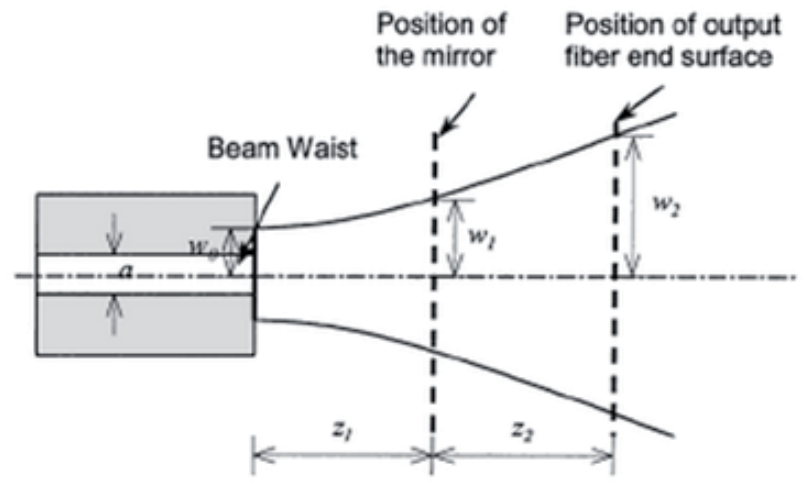

Figura 6. Divergencia del haz Gaussiano.

El haz Gaussiano procedente de la fibra se propaga en el espacio libre y el tamaño del haz está dado por:

$$
\omega=\omega_{0}\left[1+\left(\frac{\lambda z}{\pi \omega_{0}^{2}}\right)^{2}\right]^{\frac{1}{2}}
$$

Para $\omega_{0}=5.2 \mu \mathrm{m}$, el tamaño del haz en el espacio libre se muestra en la Figura 7 .

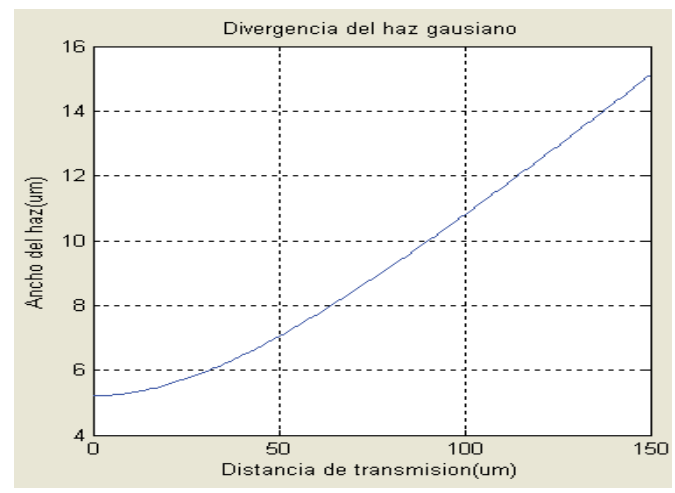

Figura 7. Ancho del haz vs Distancia de Transmisión OXC. 
Siendo, $a=0,4 \mu \mathrm{m} ; \mathrm{V}=0,87 ; \lambda=1550 \eta \mathrm{m} ; \mathrm{Z1}=0 \mu \mathrm{m}$ y $Z 2=150 \mu \mathrm{m}$. El aumento de la cintura del haz para $\omega_{1}=7.88 \mu \mathrm{m}$ y $\omega_{2}=12.95 \mu \mathrm{m}$, después de propagada es de $62,5 \mu \mathrm{m}$ y $125 \mu \mathrm{m}$, respectivamente.

Cuando dos SMF se juntan unos con otros, tres tipos diferentes de desalineaciones, llamadas longitudinales, laterales y angulares, pueden aparecer en función de la posición relativa de las dos fibras, como se muestra en la Figura 8, [10].

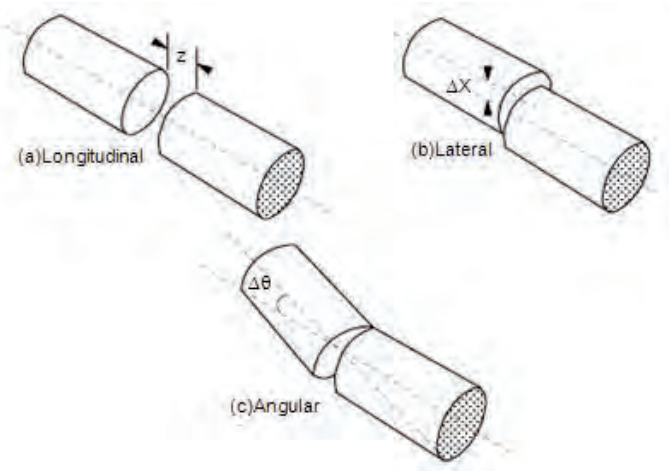

Figura 8. Desalineación entre la entrada y la salida de las fibras: a) longitudinal, b) lateral, c) angular.

Para analizar los diferentes efectos sobre las pérdidas, estos tres tipos de desalineaciones se tratan por separado [4].

Pérdida por longitud: siendo $\Delta \mathrm{x}=0$ y $\Delta \theta=0$, la relación entre la pérdida de inserción y la distancia entre los extremos de las dos fibras se puede escribir en forma de:

$$
L=-10 \log \left\{4 \cdot \frac{1}{M^{2}+4}\right\}
$$

Donde:

$$
M=\frac{\lambda * Z}{\pi \cdot n \cdot \omega_{0}^{2}}
$$

$n$ es el índice de refracción del medio, es decir, 1.0 en un espacio libre, y $\omega T=\omega R=$ $\omega 0$. La relación se muestra en la Figura 9.

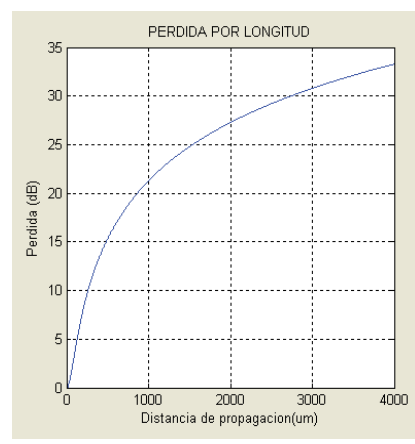

a)

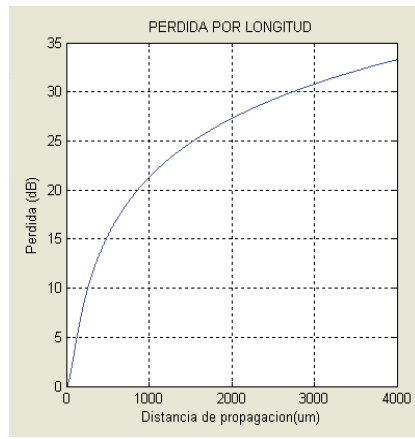

b)

Figura 9. Pérdida de acoplamiento del OXC en función de la distancia de transmisión sobre un rango de: a) 0 a $4000 \mu \mathrm{m}$ y b) 0 a $60 \mu \mathrm{m}$. 
9a. Siendo, $a=0,4 \mu \mathrm{m} ; \mathrm{V}=0,87 ; \lambda=1550 \eta \mathrm{m} ; \mathrm{Z} 1=0 \mu \mathrm{m}$ y $Z 2=4000 \mu \mathrm{m}$

9b. Siendo, $\mathrm{a}=0,4 \mu \mathrm{m} ; \mathrm{V}=0,87 ; \lambda=1550 \eta \mathrm{m} ; \mathrm{Z} 1=0 \mu \mathrm{m}$ y $Z 2=60 \mu \mathrm{m}$

Pérdida por desalineamiento lateral: siendo $\Delta z=0$ y $\Delta \theta=0$, la relación entre la pérdida de inserción y la distancia lateral entre los extremos de las dos fibras tiene la forma de:

$$
L=-10 \log \left\{\exp \left(-\frac{\Delta x^{2}}{\omega_{0}^{2}}\right)\right\}
$$

Donde $\Delta x$ es la distancia de desalineación lateral, y $\omega T=\omega R=\omega 0$. La relación entre la pérdida de energía y desalineación lateral se muestra en la Figura 10.

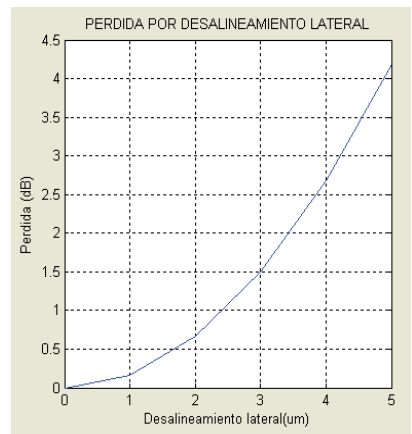

Figura 10. Pérdida de acoplamiento vs. desalineamiento lateral en el interruptor OXC.

Siendo, $a=0,4 \mu \mathrm{m} ; \mathrm{V}=0,87 ; \lambda=1550 \eta \mathrm{m} ; \mathrm{dxmin}=0 \mu \mathrm{m}$ y $\mathrm{dxmax}=5 \mu \mathrm{m}$.

La pérdida no es infinita, aunque el desplazamiento lateral es mayor que el radio de la fibra porque el ancho del haz es mayor que el radio de la fibra.

Pérdida de desalineación angular: siendo $\Delta z=0$ y $\Delta x=0$, la relación entre la pérdida de inserción y el desalineamiento angular entre los extremos de las dos fibras tiene la forma de:

$$
L=-10 \log \left\{\exp \left(-\frac{\pi n_{0} \omega_{0} \sin \Delta \theta}{\lambda}\right)^{2}\right\}
$$

Donde $\Delta \theta$ es el ángulo de desalineamiento y $\omega_{T}=\omega_{\mathrm{R}}=\omega_{0}$. La relación entre el acoplamiento de la fibra y el desalineamiento angular se muestran en la Figura 11.

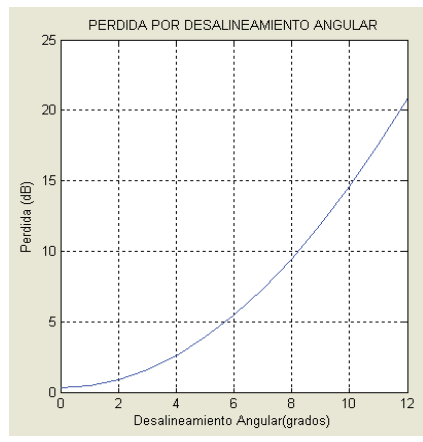

Figura 11. Pérdida por acoplamiento vs. ángulo de desalineamiento en el interruptor OXC. 
Siendo, $\mathrm{a}=0,4 \mu \mathrm{m} ; \mathrm{V}=0,87 ; \lambda=1550 \eta \mathrm{m} ; \theta \min =0^{\circ}$ y $\theta \max =12^{\circ}$

Pérdida de Fresnel: la reflexión de regreso es otra pérdida intrínseca que sucede cuando las dos fibras se acoplan. La estructura fibra-aire-fibra produce esta pérdida [4]. La pérdida de Fresnel debida a esta doble interfaz se puede calcular como:

$$
\mathrm{L}_{\mathrm{BR}}=-10 \log \left\{1-\frac{\left(\mathrm{n}_{\mathrm{F}}-\mathrm{n}_{0}\right)^{2}}{\left(\mathrm{n}_{\mathrm{F}}+\mathrm{n}_{0}\right)^{2}}\right\} \mathrm{dB}
$$

Donde $n_{F}$ y $n_{0}$ son los índices de refracción del núcleo de la fibra y el aire, que son 1.4638 y 1 , respectivamente. Por lo tanto, la pérdida de reflexión es igual a 0,1567dB por interfaz.

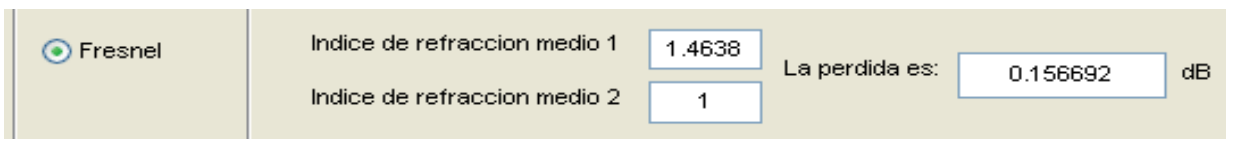

Figura 12. Pérdida de Fresnel.

\section{Pérdidas ópticas relacionadas con el microespejo}

Para la estructura del interruptor en el plano óptico, el micro-espejo es el componente que direcciona el haz. Por lo tanto, no sólo por la calidad del acoplamiento entre las fibras de entrada y salida. El rendimiento final del interruptor y el sistema óptico determinan también las propiedades ópticas de los espejos [4].

Rugosidad de la superficie: la pérdida de dispersión en la superficie del espejo está relacionada con la rugosidad superficial. La dispersión total integrada se utiliza para medir la potencia dispersa fraccional de una superficie conductora ideal suave, lisa y limpia. La potencia de dispersión debida a la rugosidad de la superficie se expresa como:

$$
\eta=1-\exp \left[-\left(\frac{4 \pi \sigma \cos \theta_{i}}{\lambda}\right)^{2}\right]
$$

Donde $\eta$ es el porcentaje de pérdida de dispersión, $\sigma$ es la raíz cuadrada media (RMS) de la rugosidad de la superficie del espejo, $\theta$ i es el ángulo de incidencia y $\lambda$ es la longitud de onda de la luz.

La pérdida en $\mathrm{dB}$, inducida por el micro-espejo se expresa como:

$$
L=-10 \log \left\{\exp \left[-\left(\frac{4 \pi \sigma \cos \theta_{i}}{\lambda}\right)^{2}\right]\right\} d B
$$

La longitud de onda $\lambda$ está determinada por el sistema de comunicación por fibra óptica y el ángulo de incidencia $\theta$ i está diseñado para ser $45^{\circ}$. La rugosidad de la superficie es un tema clave que debe considerarse durante el proceso de fabricación de un interruptor óptico MEMS de baja pérdida [4].

Material de la superficie: el reflejo del espejo es otro factor importante que influye fuertemente en la pérdida del conmutador óptico MEMS. Si el espejo está hecho de silicio mono-cristalino (SCS), la reflexión se define por la ecuación de Fresnel como: 


$$
R=\left(\frac{n_{s i}-n_{0}}{n_{s i}+n_{0}}\right)^{2}
$$

Donde $n_{\text {si }}$ y n0 son los índices de refracción del SCS y el aire, que es de 3,5 y 1,0, respectivamente.

Espesor mínimo del metal para una alta reflectividad

Un espesor específico es requerido para realizar la máxima reflexión. La reflexión no sólo depende del espesor de la capa, sino también del revestimiento metálico. La Figura 13, muestra la reflectividad en función de los diferentes metales de recubrimiento con diferentes espesores en incidencia normal. Un espejo recubierto de aluminio alcanza su máxima reflectividad del $97 \%$ cuando la película es más gruesa de $40 \mathrm{~nm}$. El oro también ofrece alta reflectividad del 97,5\% con un espesor de más de $60 \mathrm{~nm}$. Ambos metales pueden producir una mejor reflexión que el níquel y el cromo, los cuales tienen una reflectividad de 72 y $63 \%$, respectivamente [4].

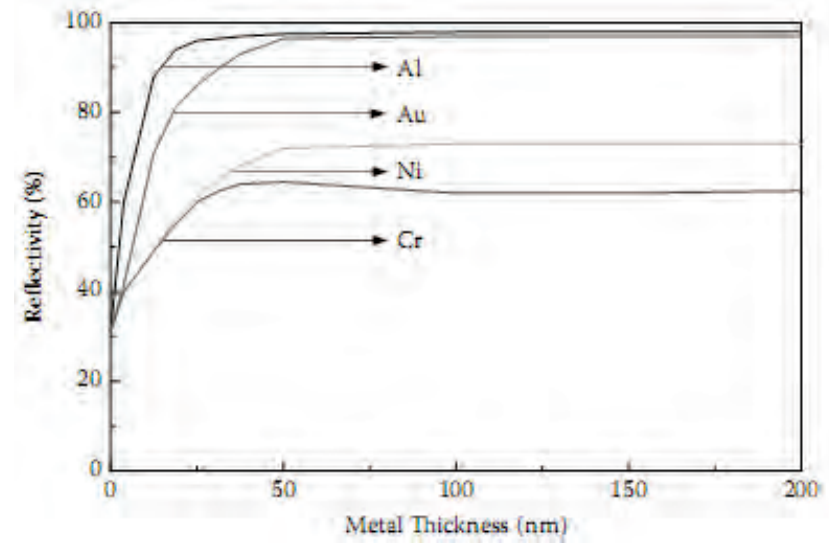

Figura 13. Reflectividad de la superficie de recubrimiento del metal en función del grosor.

Reflectividad de varias longitudes de onda: la reflectividad también varía con diferentes señales de longitudes de onda. Esta relación puede ser escrita como:

$$
\frac{1-\mathrm{R}_{1}}{1-\mathrm{R}_{2}}=\sqrt{\frac{\lambda_{2}}{\lambda_{1}}}
$$

En la longitud de onda específica de 1,55 $\mu \mathrm{m}$, la reflexión de los metales puede ser alcanzada cuando las otras longitudes de onda se conocen. En la Tabla 2 se enumeran las más altas reflexiones para cuatro metales diferentes en esta longitud de onda [12].

\begin{tabular}{|c|c|c|}
\hline Metal & $\mathbf{R}_{\mathbf{1}}\left(\boldsymbol{\lambda}_{\mathbf{1}}=\mathbf{5 8 9 3 \AA}\right)$ & $\mathbf{R}_{\mathbf{2}}\left(\boldsymbol{\lambda}_{\mathbf{2}}=\mathbf{1 5 5 0 0 \AA}\right)$ \\
\hline $\mathrm{Al}$ & 0.83 & 0.90 \\
\hline $\mathrm{Au}$ & 0.82 & 0.89 \\
\hline $\mathrm{Ni}$ & 0.66 & 0.79 \\
\hline \hline $\mathrm{Pt}$ & 0.59 & 0.75 \\
\hline
\end{tabular}

Tabla 2. Máxima reflexión de algunos metales para la longitud de onda de 1,55 $\mu \mathrm{m}$ 
Reflectividad de varios metales y longitudes de onda: en la tabla 3, se presenta el mínimo espesor necesario para que haya la máxima reflexión en algunos de los siguientes metales [12].

\begin{tabular}{|c|c|c|}
\hline Metal & Velocidad de Conmutación & Diafonía \\
\hline $\mathrm{Al}$ & 0.90 & 30 \\
\hline $\mathrm{Au}$ & 0.89 & 30 \\
\hline $\mathrm{Ni}$ & 0.79 & 200 \\
\hline $\mathrm{Pt}$ & 0.75 & 100 \\
\hline
\end{tabular}

Tabla 3. Mínimo espesor para la máxima reflexión de algunos metales.

\section{Conclusiones}

Los diferentes tipos de pérdidas de acoplamiento óptico, incluido el acoplamiento fibra a fibra, el material y el espesor de recubrimiento del espejo, se pueden utilizar para diseñar y evaluar el rendimiento óptico de los dispositivos MEMS ópticos.

Para comprobar su capacidad se ha realizado el modelo de un elemento conmutador y simulado su comportamiento en la herramienta MATLAB) y se ha pensado como base para crear un sistema aplicable a una red óptica de nueva generación. Se ha conseguido obtener un módulo conmutador OXC 2x2 basado en esta tecnología, que además de los diseños desarrollados en este proyecto, podrá ser empleado en cualquier otra simulación en la misma herramienta con su código modular. Se ha escalado con el propósito de formar una estructura de conmutación OXC $4 \times 4$ y del mismo modo, obtener un enrutador en longitud de onda (OADM) que permita extraer los canales de tráfico en las salidas deseadas.

En la demostración del buen funcionamiento del sistema se han simulado diversas situaciones, tanto para un conmutador OXC 2x2, OXC 4x4 y un OADM con óptimos resultados. Los diseños de los diferentes escenarios facilitan la ampliación y creación de nuevas simulaciones empleando esta tecnología, y los resultados obtenidos ofrecen una visión representativa del comportamiento del dispositivo recreado, que serán una parte importante en el entendimiento por parte de los alumnos de una clase impartida sobre esta tecnología en un aula de clases.

Los alcances de las simulaciones confirman los resultados analíticos y numéricos del comportamiento de los dispositivos de conmutación óptica. No obstante los avances a largo plazo de este tipo de tecnología puede abrir paso a nuevos diseños y desafíos en el campo de las redes ópticas.

\section{Referencias}

[1] J.R. Vacca, Optical networking best practices hadbook. Canada: John Wiley \& Sons, Inc. Wiley, November 28, 2006, pp. 1-3, 78-96, 263-368.

[2] G. Bernstein, B. Rajagopalan, S.Debanjan, Optical Network Control: Architecture, Protocols and Standards. USA. Pearson Edition, Inc. July 24, 2003, pp. 9-15. 
[3] A. Vivek. Optical Network Design and Implementation. USA Cisco Press. March 17, 2004. 13-38 p.

[4] A.Q. Liu. Photonic MEMS Devices Design, Fabrication and Control. Rochester, New York, USA. CRC Press Taylor and Francis Group. September 24, 2001.

[5] G.M. Rebeiz. "RF MEMS Theory, Design, and Technology» USA. New Jersey: John Wiley \& Sons, Inc. 2003. DOI: $10.1002 / 0471225282$.

[6] J. Li, A.Q. Liu, W.D. Zhong, Q.X. Zhang, C. Lu. «MEMS switch based serial reconfigurable OADM». IEEE Journal of Selected Areas in Communications: Optics Communications USA 2004, 83 p. [Job grade]. Nanyang Technological University, Institute of Microelectronics Singapore. School of Electrical and Electronic Engineering.

[7] I.P. Kaminow, T. Li, A.E. Willner. Optical Fiber Telecommunications. Fifth Edition: Components and Subsystems. Elsevier Inc. 2008. 714 p.

[8] M.C. Wu, F.O. Solgaard, J. E. Ford. «Optical MEMS for Lightwave Communication». IEEE Journal of Lightwave Technology, Vol. 24, No. 12, December 2006.

[9] K.R. Cochran, L. Fan, D. L. DeVoe. "Moving reflector type micro optical switch for high-power transfer in a MEMS-based safety and arming system». IEEE Journal of Micromechanics and Micro-engineering 2003. 139 p. [Job grade]. Department of Mechanical Engineering, University of Maryland College Park, Indian Head Division, Naval Surface Warfare Center, Indian Head, MD.

[10] J. Li, Q.X. Zhang, A.Q. Liu. "Advanced fiber optical switches using deep RIE (DRIE) fabrication». IEEE Journal of Selected Topics in Quantum Electronics 2002. [Job grade]. School of Electrical \& Electronic Engineering, Nanyang Technological University, Institute of Microelectronics.

[11] G. Farrell. "Connector Misalegnment Loss». Optical Communications Laboratory, Exercise No.7. 2002. p. 3.

[12] M. F. Volodymyr, V. B. Yaroslav. «High transmission of system «dielectric grating, thin metal film dielectric grating" 2004. Lviv Polytechnic National University, Institute of Technology Rzeszow University. Dep. of Fotonics.

[13] M. Xie, X. Wang, M. Yu, M. Zhang, G. Wang. Analysis of the air damping in MEMS lateral driven microresonators. In: Proceedings of the ninth international IEEE CPMT symposium on high density design, packaging and microsystem integration (HDP'07); 2007. p. 1

[14] T. Yamamoto, J. Yamaguchi, N. Takeuchi, A. Shimizu, E. Higurashi, R. Sawada, and Y. Uenishi, «A three-dimensional MEMS optical switching module having 100 input and 100 output ports» IEEE Photon. Technol. Lett., vol. 15, no. 10, pp. 1360-1362, Oct. 2003.

[15] X. Zheng, V. Kaman, Y. Shifu, X. Yuanjian, O. Jerphagnon, K. Adrian, R. C. Anderson, H. N. Poulsen, L. Bin, J. R. Sechrist, C. Pusarla, R. Helkey, D. J. Blumenthal, and J. E. Bowers, "Three-dimensional MEMS photonic cross-connect switch design and performance," IEEE J. Sel. Topics Quantum Electron, vol. 9, no. 2, pp. 571-578, Mar./Apr. 2003.

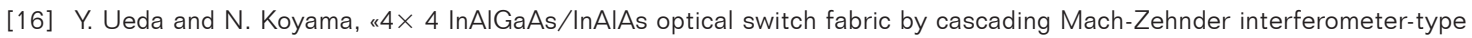
optical switches with low-power and low-polarization-dependent operation," Compound Semiconductor Week (CSW/ IPRM), vol. 24, no. 9, pp. 757-759, 2011.

[17] T. Yeow, «MEMS optical switches,» Communications Magazine, IEEE, November, pp. 158-163, 2001.

[18] L. Savastano and G. Maier, "Physical-parameter design in 2-D MEMS optical switches, Lightwave Technology, Journal of, vol. 23, no. 10, pp. 3147-3155, 2005.

[19] M. I. Anis, N. Ahmed, and U. Ahmed, "Performance analysis of optical network based on OADM by using different filters," in Application of Information and Communication Technologies, AICT 2009. International Conference on, 2009, pp. $1-5$.

[20] J. Yuefeng, J. Zhang, S. Yongmei, G. Wanyi, Y. Bing, Z. Yong, «Research and realization of OADM technology in metro optical network» Proceedings Vol. 453, Metro and Acess Networks, Wanyi Gu; Jianhui; Jin-Yi Pan, Editors, pp.159-170, Publication: SPIE.org, 16 oct 2001.

[21] S. Singh and L. Kumar, "Performance Investigations on DWDM-OADM Optical Ring Network for CRZ Data Format at Different Fiber Length,» International Journal of Scientific \& Engineering Research, vol. 2, no. 9, pp. 1-8, 2011.

[22] G. Barozzi, M. Lo Papa, and F. Meli, "Configurable OADM based on novel tunable Bragg grating," Lasers and ElectroOptics Society 2000 Annual Meeting. LEOS 2000. 13th Annual Meeting. IEEE, vol. 2, no. I, pp. 549-550, 2000.

[23] C. Y. Li, G. M. Li, V. O. K. Li, P. K. a. Wai, H. Xie, and X. C. Yuan, "Using 2x2 switching modules to build large 2-D MEMS optical switches," GLOBECOM '03. IEEE Global Telecommunications Conference (IEEE Cat. No.03CH37489), vol. 5, pp. 2798-2802, 2003.

[24] P. Hlen, "Noise and crosstalk limitations in optical cross-connects with reshaping wavelength converters," Journal of lightwave technology, vol. 17, no. 8, pp. 1294-1301, 1999. 
[25] G. Shen, T. Cheng, and S. Bose, "Architectural design for multistage 2-D MEMS optical switches," JOURNAL OF LIGHTWAVE TECHNOLOGY, vol. 20, no. 2, pp. 178-187, 2002.

[26] G. Zhu and G. Kuo, "A Novel Integrated Multistage 2-D MEMS Optical Switch With Spanke-Benes Architecture," Journal of Lightwave Technology, vol. 26, no. 5, pp. 560-568, 2008.

[27] G. G. S. Kuo, «INTEGRATED MULTISTAGE MEMS-BASED OPTICAL SWITCH,»Symposium on Design, Test, Integration and Packaging of MEMS/MOEMS 2003., no. May, pp. 113-116, 2003.

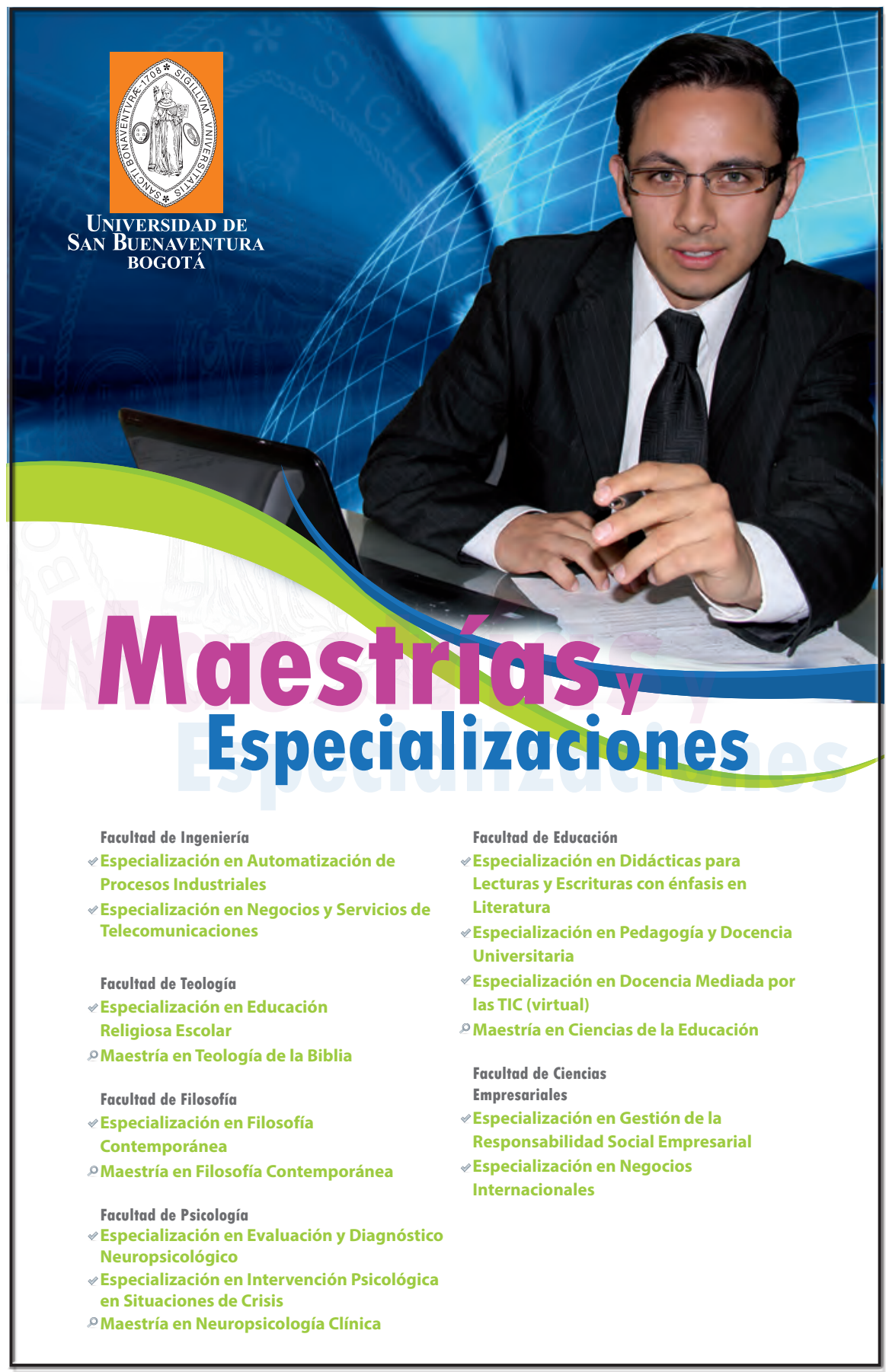

